\title{
Characteristics of bicycle travel in Japan and the basic concept of the Bicycle Travel Space Development Guideline
}

\author{
H. Kobayashi ${ }^{1,2}$, H. Honda ${ }^{2}$ \& H. Yoshida ${ }^{3}$ \\ ${ }^{1}$ Kyoto University, Japan \\ ${ }^{2}$ National Institute for Land and Infrastructure Management, \\ Ministry of Land, Infrastructure, Transport and Tourism, Japan \\ ${ }^{3}$ University of Tokyo, Japan
}

\begin{abstract}
In 2012, the Ministry of Land, Infrastructure, Transport and Tourism (MLIT) and the National Police Agency (NPA) issued the "Guideline for the Creation of Safe and Comfortable Bicycle Use Environments in Japan" to road managers and to prefectural police in charge of road traffic administration in their respective jurisdiction. The Guideline was prepared jointly by MLIT and NPA based on the results of surveys and research by the National Institute for Land and Infrastructure Management (NILIM), and presented concepts to guide the creation of environments for using bicycles throughout Japan in the future.

This paper explains the characteristics of bicycle travel in Japan and presents an outline and the concepts of Japanese bicycle guideline, which states principles for the provision of road space to allow bicycles to travel safely and comfortably on roads.

The Guideline is divided into four chapters: Planning, Design, Compliance with Rules, and Overall Initiatives, and the concepts and points of the Guideline especially "Planning" are outlined in this paper. The "Planning" chapter presents planning and preparation procedures, and detailed methods for studying the creation of a bicycle network plan, in order to effectively create efficient spaces for travelling by bicycle. The "Design" chapter outlines precautions when designing bicycle travelling spaces for uninterrupted roads and for intersections.

Keywords: bicycle guideline, bicycle network plan, bicycle travelling space creation.
\end{abstract}




\section{Characteristics and challenges of bicycle travel in Japan}

In Japan, the number of bicycles owned in Japan tends to increase as in the case of car ownership since the 1970s in which the statistic data exist. As of 2008, a total of 69 million bicycles were owned by Japanese residents, which is estimated to be about 1.3 bicycles owned by each household. Nationwide average 11.6\% (2010) (in Osaka, 28\%, and in Tokyo 23 wards, 16\%) of these people use their bicycles to commute to work or school. Thus, the number of cyclists in Japan is higher than in other countries, and bicycles are an important and convenient means of urban transportation.

For example, the percentages of road users by method of travelling by distance traveled are shown in Figure 1, revealing that about $20 \%$ of trips of less than $5 \mathrm{~km}$ are done by bicycle. This indicates that when travelling up to about 5 $\mathrm{km}$, the bicycle is quick and easy. The reason of a high ratio of less than $5 \mathrm{~km}$ trips would be the bicycle being not only an easily accessible means of transportation but also a faster means of transportation for a less than $5 \mathrm{~km}$ trip than other means of transportation such as car or railroad.

In response to growing awareness of health and the environment, the need to cycle is rising while the purposes of using bicycles are diversifying. For example, Figure 2 shows the results of a questionnaire survey on the reasons for using bicycles: many people report that they cycle because it is healthy and economical. In Japan, the sales of sports type bicycles, such as what is called "road cycles", (sales volume increased by about $380 \%$ from 2003 to 2010) or electric bicycles (sales volume increased by about $170 \%$ from 2003 to 2010) are increasing in market share in these years, indicating diversification of bicycle use. Of all age brackets, the women aged 60 years or older and the men aged 60 years or older occupy $28 \%$ and $21 \%$ of all bicycle users, respectively. This indicates a high ratio of bicycle users even among the elderly. Furthermore, seniors with weaker leg power can casually ride bicycles as a result of the spread of power-assisted bicycles. Those bicycles are considered to help the elderly expand their range of action or go out more often. Considering this, use of bicycles has a big significance in the future.

On the other hand, although the total number of traffic accidents has fallen in recent years, as shown in Figure 3, the percentage of accidents involving bicycles is increasing. Bicycle/pedestrian accidents are rising particularly rapidly, and have increased by about 1.5 times in the past 10 years. Rules governing cycling have not fully penetrated. Figure 4 shows the state of compliance with cycling rules; it seems that many people do not comply with the rules even though they are aware of them. There are some cases observed where bicycle users act out of selfishness, flexibly using roadways and sidewalks depending on their recognition. These users travel roadways as vehicles as they like but suddenly move to sidewalks depending on the traffic lights. 


\section{Past initiatives concerning bicycle countermeasures}

In response to the soaring number of traffic accidents accompanying motorization in Japan since the 1960s, traffic regulations were introduced which permitted cyclists to use sidewalks provided they travel at speeds and in a manner which do not obstruct the movement of pedestrians, thereby separating bicycles and automobiles.

However, cyclists became less aware that bicycles are vehicles, and increasingly rode their bicycles in a dangerous manner for pedestrians by not obeying cycling rules on sidewalks. This made it difficult to establish countermeasures to prevent accidents between bicycles and pedestrians.

In January 2008, Ministry of Land, Infrastructure, Transport and Tourism (MLIT) and National Police Agency (NPA) designated 98 districts throughout Japan as "Model Districts for Improving the Bicycle Travelling Environment" in order to install bicycle tracks and lanes. In October 2011, NPA announced comprehensive countermeasures to ensure the safety of bicycle users and pedestrians, under the basic principle that bicycles are vehicles.

In response, beginning in November 2011, MLIT and NPA set up a committee to study the creation of environments for safe and comfortable bicycle use. In April 2012, the committee presented MLIT and NPA with a "Proposal for Bicycle Environments for the Benefit of All - the Creation of Safe and Comfortable Bicycle Use Environments." Incidentally, the Interim Draft of the Proposal by the Road Subcommittee, Council for Social Infrastructure of June 2012, included the provision of environments which "diverse users can share safely and securely", including users of bicycles and vehicles other than automobiles in order to transform road policy.

The Guideline for the Creation of Safe and Comfortable Bicycle Use Environments in Japan introduced by this paper was prepared based on a proposal made by the committee in April 2012. The Guideline is intended to be used by regional road managers or prefectural police to prepare plans for bicycle networks and to encourage the establishment of such networks while ensuring compliance with bicycle travel rules.

The Guideline contains items which should be implemented cooperatively with river managers, port managers, and other administrative bodies, local residents, road users and other persons involved in creating bicycle environments, to permit a wide range of both 'soft' and 'hard' initiatives accompanying the creation of bicycle travelling space while ensuring that all road users strictly comply with bicycle travel rules. It also states that various initiatives should be studied by appropriately sharing roles among relevant organizations based on regional circumstances.

The Guideline summarizes practical items to be studied concerning bicycle network routes selected according to the basic principles or planning goals for bicycle network planning. It also stipulates that other routes should be considered.

The Guideline presents standard concepts, and indicates that specific initiatives should be based on regional challenges and needs, and on the state of 
traffic. The Guideline will be revised regularly in line with emerging new technical knowledge or changes to the legal system.

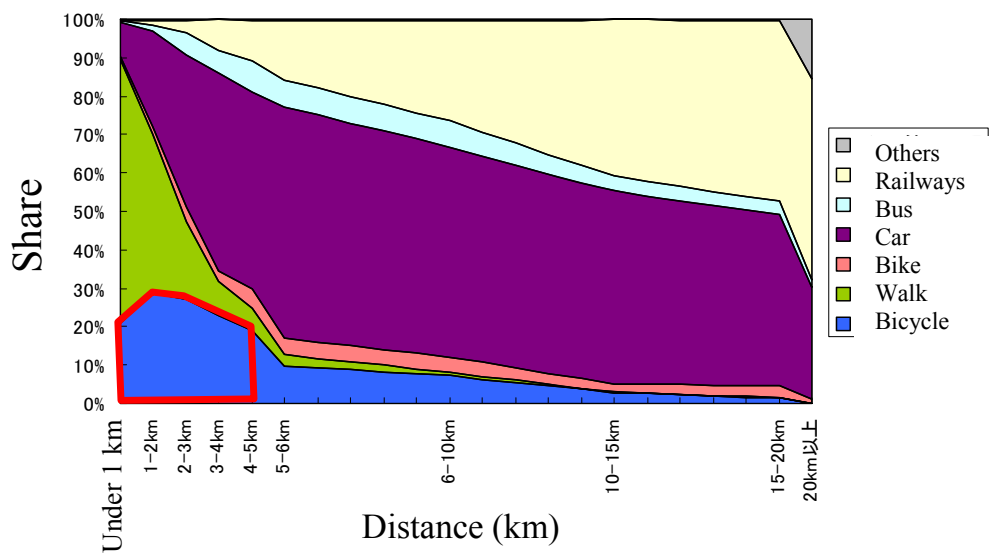

Figure 1: Percentages of travel modes by distance travelled [1].

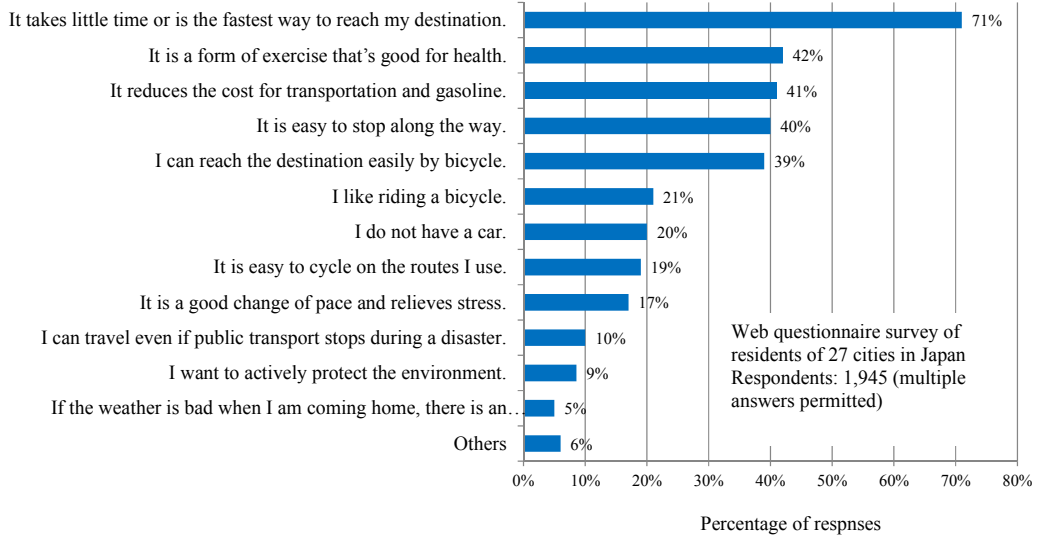

Figure 2: Reasons for using bicycles.

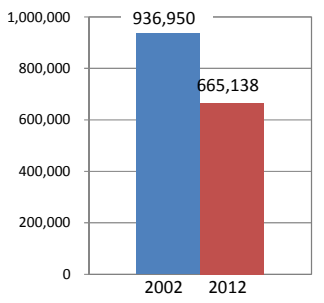

Total traffic accidents

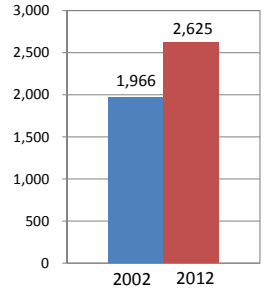

Bicycle/Pedestrian accidents

Figure 3: Recent trends in bicycle/pedestrian accidents [2]. 


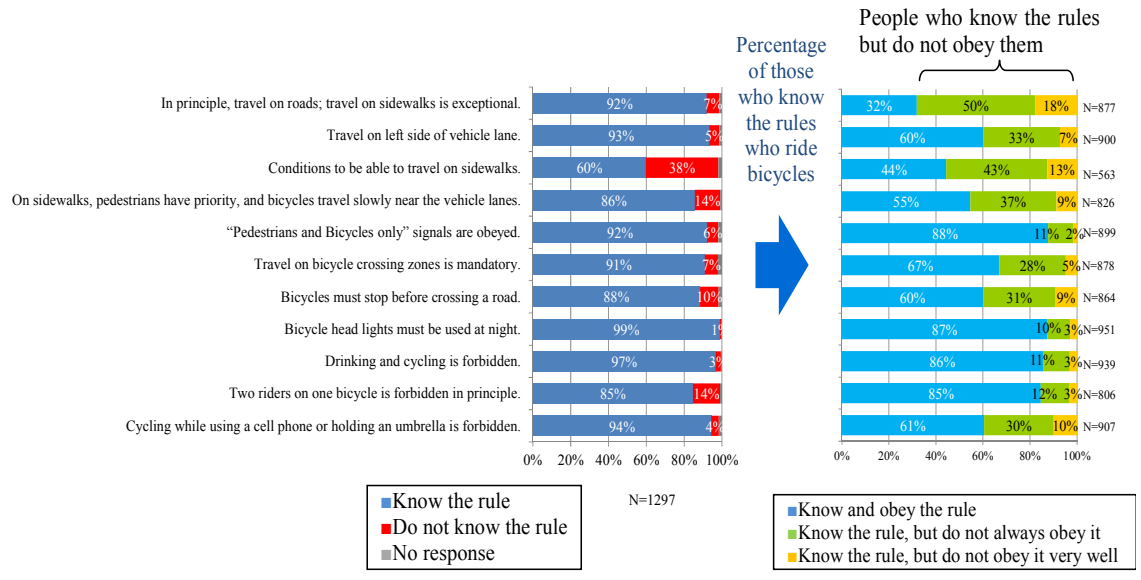

*Prepared based on the results of a questionnaire survey of 1,297 visitors to driving license test centers, etc. nationwide conducted by the National Police Agency in October 2011.

Figure 4: Compliance with bicycle travel regulations.

\section{Positioning of the Guideline}

The Guideline for the Creation of Safe and Comfortable Bicycle Use Environments in Japan introduced by this paper was prepared based on a proposal made by the committee in April 2012. The Guideline is intended to be used by regional road managers or prefectural police to prepare plans for bicycle networks and to encourage the establishment of such networks while ensuring compliance with bicycle travel rules.

The Guideline contains items which should be implemented cooperatively with river managers, port managers, and other administrative bodies, local residents, road users and other persons involved in creating bicycle environments, to permit a wide range of both 'soft' and 'hard' initiatives accompanying the creation of bicycle travelling space while ensuring that all road users strictly comply with bicycle travel rules. It also states that various initiatives should be studied by appropriately sharing roles among relevant organizations based on regional circumstances.

The Guideline summarizes practical items to be studied concerning bicycle network routes selected according to the basic principles or planning goals for bicycle network planning. It also stipulates that other routes should be considered.

The Guideline presents standard concepts, and indicates that specific initiatives should be based on regional challenges and needs, and on the state of traffic. The Guideline will be revised regularly in line with emerging new technical knowledge or changes to the legal system. 


\section{Outline and concept of the Guideline}

The Guideline is divided into four chapters: Planning, Design, Compliance with Rules, and Overall Initiatives. The concepts and points of the Guideline are outlined below.

\subsection{Planning bicycle travelling space}

This chapter presents planning and preparation procedures, and detailed methods for studying the creation of a bicycle network plan, in order to effectively create efficient spaces for travelling by bicycle.

Figure 5 shows the procedure for creating a bicycle network plan. First, the state of bicycle use and problems are clarified, and basic principles and goals for the bicycle network plan are set in line with regional upper-level plans and related plans. Next, bicycle network routes necessary to achieve those goals are selected. This is done in order to effectively create efficient spaces for travelling by bicycle in line with the goals, because it is unrealistic to create bicycle travelling space on all roads. The Guideline also presents the perspective of selected routes and the criteria for network density as reference information.

On the selected routes, the major principle is that bicycles should travel on vehicle roads. To improve safety for bicycles when travelling on vehicle roads, the need to separate bicycles from automobiles is studied based on the state of traffic on the route (speed and volume of automobile traffic) to select the type of bicycle travelling space. The following points are relevant:

1) The Guideline presents three patterns for bicycle travelling space: bicycle tracks, bicycle lanes, and mixed use on vehicle lanes by bicycles and automobiles (mixed-vehicle lanes) (Figure 6).

2) The Guideline also presents criteria for assessing the state of traffic (speed and volume of automobile traffic), as a reference for studying the need for separation (Figure 7).

A bicycle track is a track demarcated by curbstones, etc. for travel by bicycles. It can be one-way, but is generally two-way. A bicycle lane is a vehicle travelling lane used only by bicycles, and the space is visually demarcated by color or line from the lanes used by automobiles. Because it is a vehicle travel lane, bicycles can only travel in the same direction as automobiles, and so the lane is a one-way travelling space. Mixed use on vehicle lanes refers to space that is shared by bicycles and automobiles on the same vehicle lane, and they are basically not separated. To improve safety, road shoulders are colored or belt-shaped road surface markings or pictograms are often placed.

The criteria for the state of traffic, which is reference information used to study the need for separation, are comprehensively judged according to technical standards in other countries [3-8] and the state of improvement in Japan.

Application of the type of bicycle travelling space selected for existing road spaces is studied based on the following concepts: 


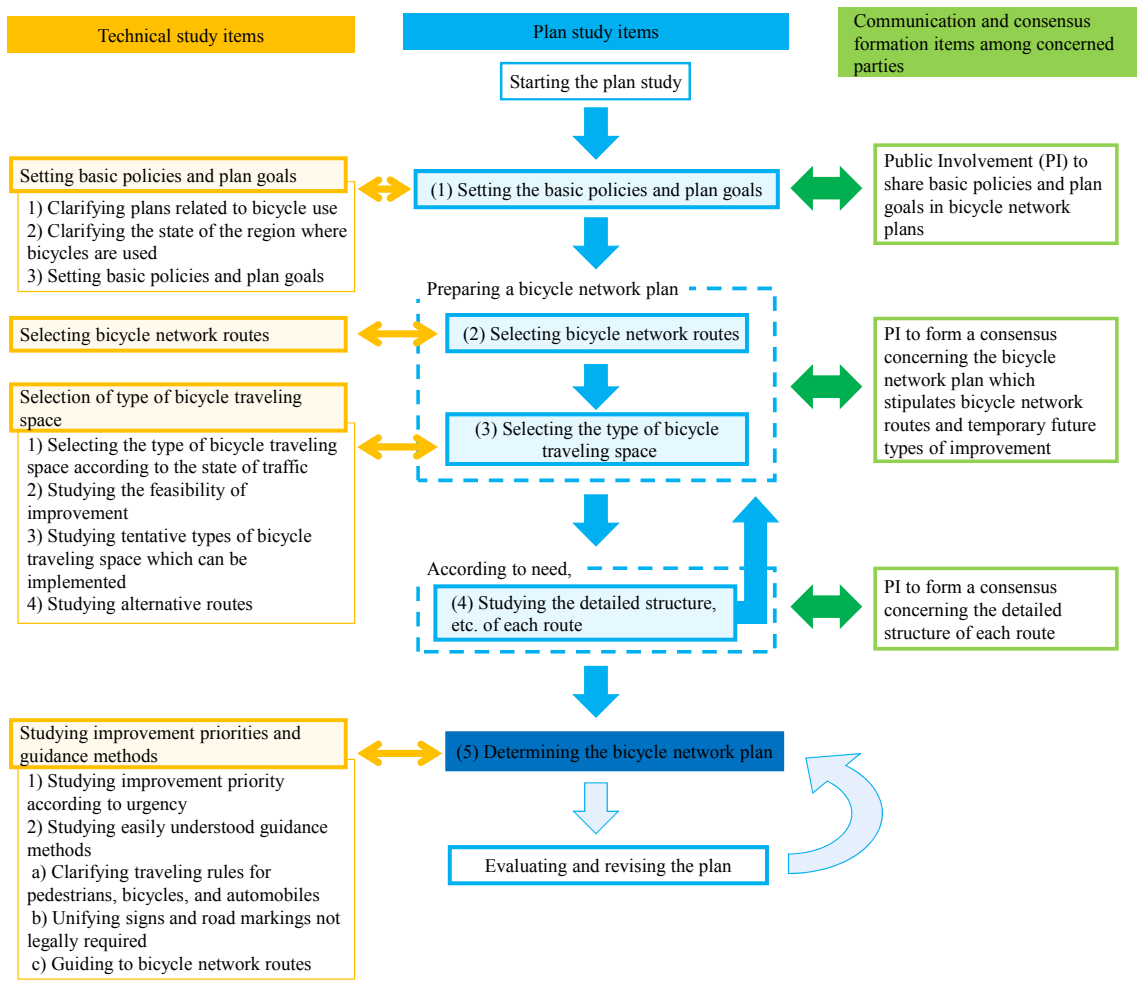

Figure 5: Procedure for creating a bicycle network plan.

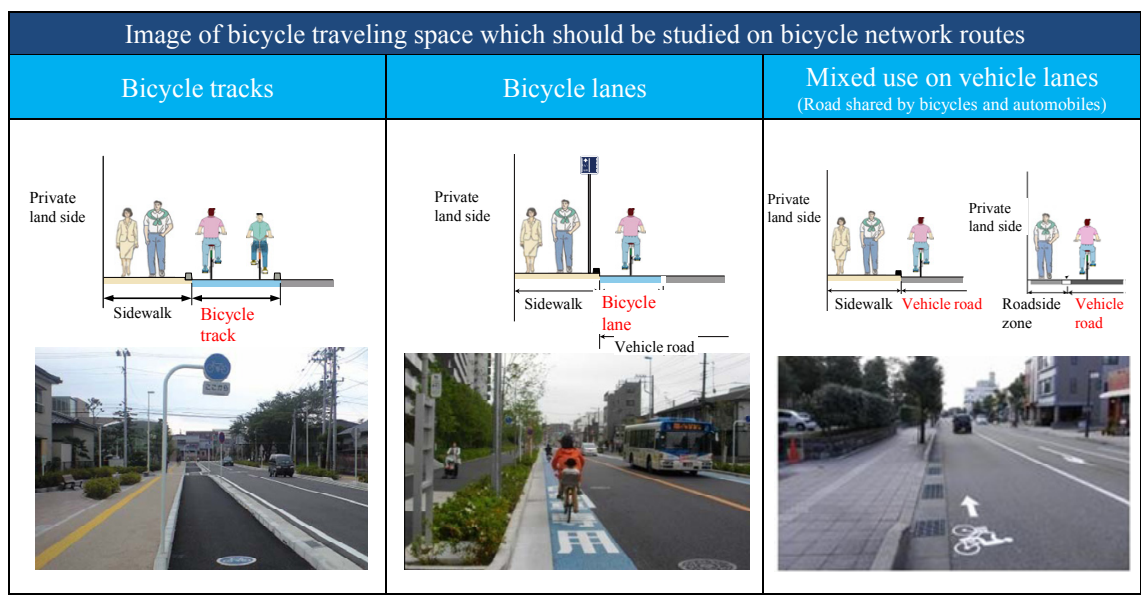

Figure 6: Image of bicycle travelling spaces on bicycle network routes. 


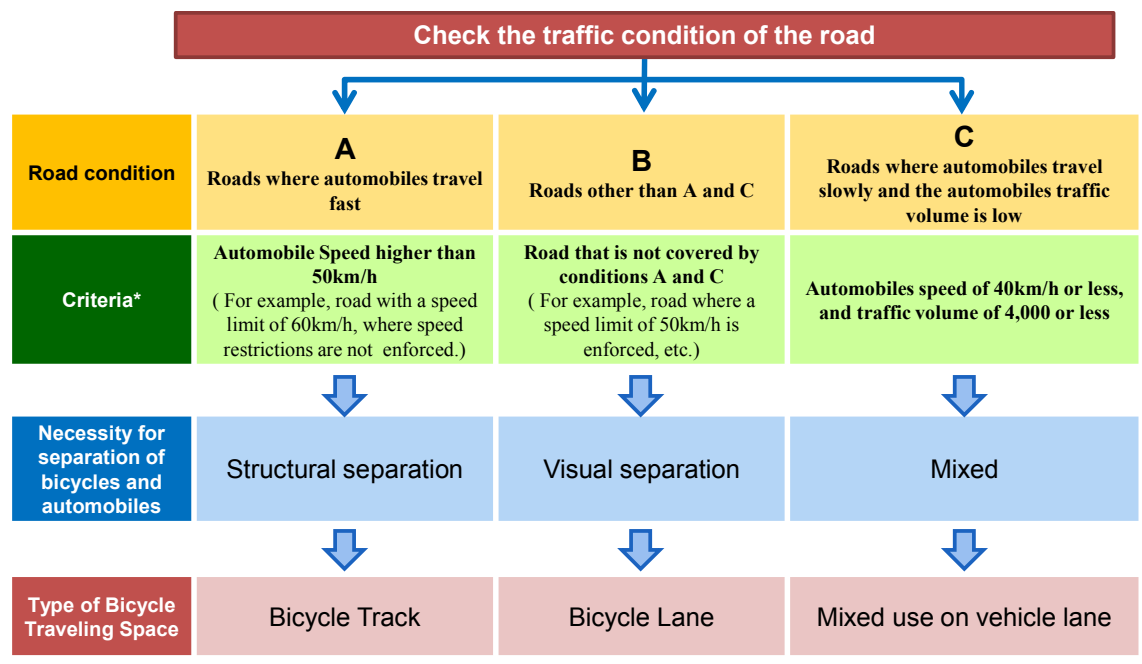

* This is a mere reference value. The necessity of separation may be reviewed individually for each area depending on the traffic condition and other factors.

Figure 7: Type of improvement of bicycle travelling space according to the state of traffic.

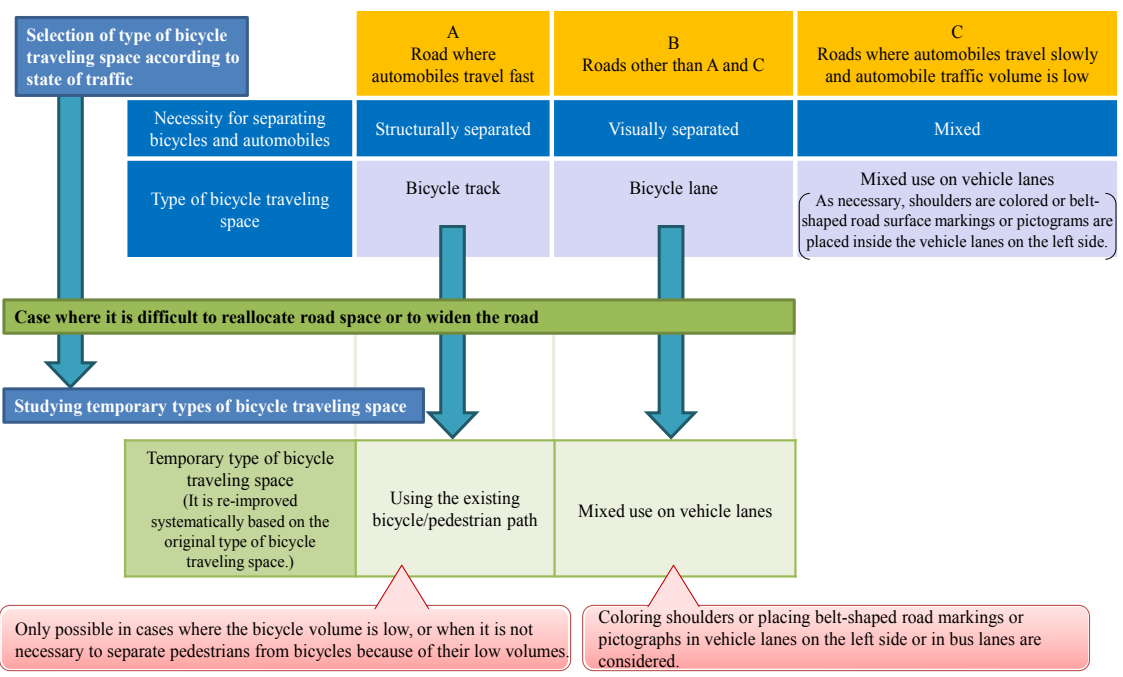

Figure 8: Concept of selecting feasible temporary type of bicycle travelling space. 
1) The widths of each part of a road - vehicle lanes, center median, road shoulders, parking lanes, sidewalks, side strips, green belts, etc. - are reviewed, to study the reallocation of the road space.

2) Reducing the number of vehicle lanes or changing the road to one-way by providing new roads nearby or managing traffic demand to restrict automobile traffic on the road are studied.

3) On roads where automobile speed should be restricted, enforcing speed limits and revising the type of bicycle travelling space itself is studied.

If the results of these studies show that it will be difficult to apply the selected type of bicycle travelling space, a temporary type of bicycle travelling space or alternative route may be considered. The Guideline stipulates that instead of abandoning the improvement because it is difficult, priority must be placed on promptly creating a bicycle network by studying temporary types of bicycle travelling space, even if they are not fully effective. Figure 8 presents the concept of temporary bicycle travelling spaces. If it is difficult to create a bicycle track, an existing bicycle/pedestrian path can be considered. If it is difficult to provide a bicycle lane, mixed travel by bicycles and automobiles on a vehicle lane can be considered. However, continuing to use an existing bicycle/pedestrian path should only be permitted when the bicycle volume is low, or when it is not necessary to separate pedestrians from bicycles because of their low volumes. When mixed travel on vehicle lanes is adopted, it is essential to clearly mark the route for bicycles.

These studies for creating a bicycle network plan must be carried out in cooperation with the road manager, police and other administrative bodies in each region, and with a broad range of local residents and other parties.

\subsection{Design of bicycle travelling space}

This chapter outlines precautions when designing bicycle travelling spaces for uninterrupted roads and for intersections. The following are key considerations presented in the Guideline.

\subsubsection{Design for uninterrupted road sections}

1) Bicycle tracks

- The Guideline presents concepts of separation structures (in the case of structural separation, installing curbstones instead of guard fences).

- The Guideline presents concepts of deciding widths or conditions requiring widening, etc. (for a bicycle track, $2 \mathrm{~m}$ or more, etc.).

2) Bicycle lanes

- The Guideline presents how to deal with a one-way road excluding bicycles (contra-flow roads), etc.

3) Mixed use by bicycles and automobiles on a vehicle lane

- The Guideline presents the concept of width in a case where the shoulders are colored or there are belt-shaped road markings, etc. It also presents design precautions for sections with bus stops, pedestrian bridges, parking meters and other structures. 


\subsubsection{Design for intersections}

The concept of intersection design is as follows.

1) At intersections, separation form must maximize continuity of similar forms of bicycle travelling spaces on uninterrupted roads. The bicycle travelling space is not easily connected to bicycle/pedestrian paths.

2) With priority on the straightness of the line of motion of bicycles, bicycle travelling positions at intersections of bicycles travelling in the same direction as automobiles are linked linearly (one-way bicycle tracks and bicycle lanes).

3) To inform automobile drivers, etc. of the line of motion of bicycles, road surface markings are placed to show where and how bicycles travel.

4) To prevent accidents between bicycles and automobiles turning left at the entrances to intersections, one of the following two methods is selected. The Guideline shows the strengths and weaknesses of the two methods and precautions for each method, and stipulates that the method should be selected according to local conditions.

Method (1) "Discontinuing the bicycle track or bicycle lane just before the intersection, widening the vehicle lane on the left side of the road and placing road surface markings to show the bicycle travelling position so that bicycles and automobiles turning left travel together in a line."

Method (2) "Connecting bicycle tracks or bicycle lanes at the intersection to separate the bicycles from automobiles."

5) Showing where bicycles should stop at an intersection when making a twostep right turn.

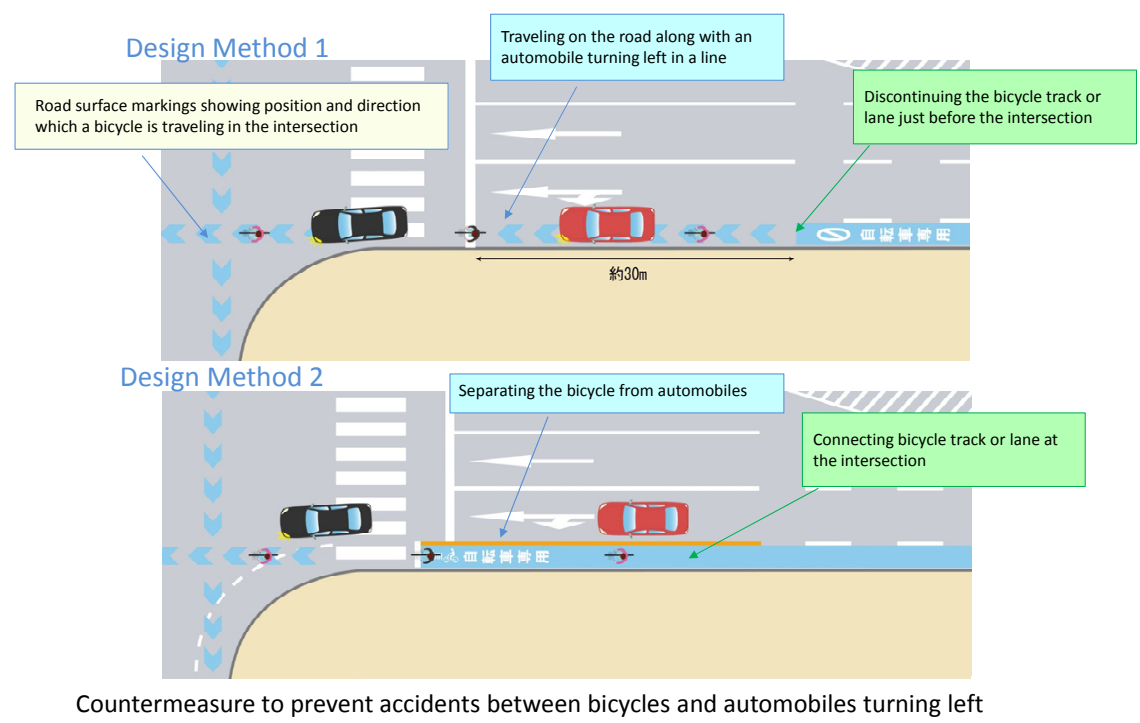

Figure 9: Examples of design of Bicycle Travelling Space at a four-leg intersection. 
When creating bicycle travelling space at intersections on narrow roads and it would be difficult to ensure space even by reducing vehicle lane width, first, road markings to show the bicycle travelling position and travelling method are placed on the road, then method (3) is implemented, introducing mixed use by bicycles and automobiles on a vehicle lane.

The Guideline also lists precautions when designing measures for intersections with narrow streets, three-leg intersections and intersections with left-turn lanes formed by median strips.

Education on the rules of how to use
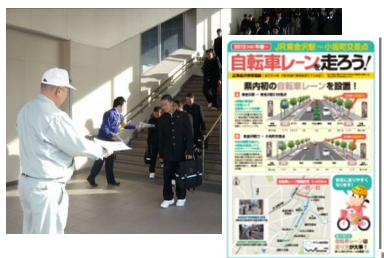

Distribution of flyers with the help of local residents
Provision of incentives to compliance with the rules

Guidance and control of traffic regulation violators

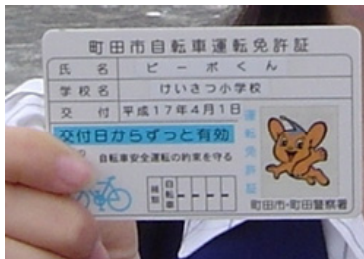

Distribution of bicycle license at elementary school

Figure 10: Thorough education on use rules (case example).

Measures to cope with parked and loading/unloading vehicles

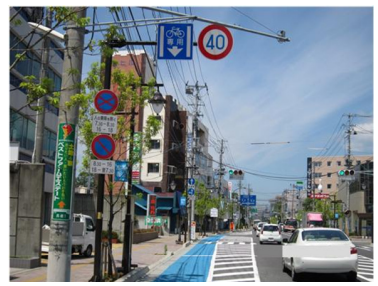

Banning of parking along the road in the commuting time zone

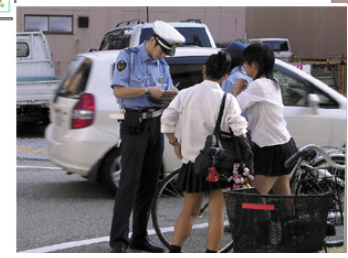

Guidance and control on use of bicycles

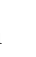

的




\subsection{Compliance with rules of use}

It is necessary to make sure that not only cyclists, but also all road users including pedestrians and automobile drivers understand that a bicycle is a vehicle, and to make sure that they comply with all rules including cycling rules, bicycle parking rules and automobile parking rules. This chapter presents how to educate all road users of the rules, give them incentives to obey the rules, and implement guidance and enforcement to prevent traffic violations such as Figure 10 .

\subsection{Overall initiatives concerning bicycle use}

This chapter presents two kinds of bicycle use initiatives: those intended to effectively use bicycle travelling space and those intended to promote the use of bicycles. Regarding the former, the Guideline presents countermeasures for parked cars and unloading trucks, as well as discarded bicycles, to resolve the trade-off relationship between ensuring bicycle travelling space and automobile parking. Regarding promoting the use of bicycles, the Guideline includes information about safety and enjoyable use as well as initiatives to meet regional needs and challenges related to health, the environment, and the promotion of tourism such as Figure 11.

\section{Conclusions}

The Guideline is a useful source of information for creating regional bicycle network plans and creating safe and comfortable bicycle travelling spaces in Japan. The Guideline will be revised based on technical knowledge obtained in future and revisions of legal standards. The National Institute for Land and Infrastructure Management will also conduct technical studies on the application of the Guideline throughout Japan.

\section{References}

[1] National Urban Traffic Characteristics Survey in 2005, Japan, 2005

[2] State of traffic accidents in 2011, National Police Agency, Japan, 2011

[3] Design manual for bicycle traffic, CROW, Netherland, 2006

[4] Empfehlungen für Radverkehrsanlagen, FGSV, Germany, 2010

[5] Recommandations pour les aménagements cyclables, Certu, France, 2008

[6] Guide for the Development of Bicycle Facilities, AASHTO, USA., 2012

[7] Cycling Aspects of Austroads Guides, Austroads, Australia, 2011

[8] London Cycling Design Standards, Transport for London, 2005 